\title{
Core concepts for dynamic TU games
}

Citation for published version (APA):

Peters, H. J. M., \& Perea ý Monsuwé, A. (2001). Core concepts for dynamic TU games. METEOR, Maastricht University School of Business and Economics. METEOR Research Memorandum No. 024 https://doi.org/10.26481/umamet.2001024

Document status and date:

Published: 01/01/2001

DOI:

10.26481/umamet.2001024

Document Version:

Publisher's PDF, also known as Version of record

\section{Please check the document version of this publication:}

- A submitted manuscript is the version of the article upon submission and before peer-review. There can be important differences between the submitted version and the official published version of record.

People interested in the research are advised to contact the author for the final version of the publication, or visit the DOI to the publisher's website.

- The final author version and the galley proof are versions of the publication after peer review.

- The final published version features the final layout of the paper including the volume, issue and page numbers.

Link to publication

\footnotetext{
General rights rights.

- You may freely distribute the URL identifying the publication in the public portal. please follow below link for the End User Agreement:

www.umlib.nl/taverne-license

Take down policy

If you believe that this document breaches copyright please contact us at:

repository@maastrichtuniversity.nl

providing details and we will investigate your claim.
}

Copyright and moral rights for the publications made accessible in the public portal are retained by the authors and/or other copyright owners and it is a condition of accessing publications that users recognise and abide by the legal requirements associated with these

- Users may download and print one copy of any publication from the public portal for the purpose of private study or research.

- You may not further distribute the material or use it for any profit-making activity or commercial gain

If the publication is distributed under the terms of Article $25 \mathrm{fa}$ of the Dutch Copyright Act, indicated by the "Taverne" license above, 


\title{
Core Concepts for Dynamic TU Games
}

\author{
Laurence Kranich* $^{*}$ Andrés Perea ${ }^{\dagger}$ Hans Peters ${ }^{\ddagger}$
}

December 2001

\begin{abstract}
This paper is concerned with the question of how to define the core when cooperation takes place in a dynamic setting. The focus is on dynamic cooperative games in which the players face a finite sequence of exogenously specified TU-games. Three different core concepts are presented: the classical core, the strong sequential core and the weak sequential core. The differences between the concepts arise from different interpretations of profitable deviations by coalitions. Sufficient conditions are given for nonemptiness of the classical core in general and of the weak sequential core for the case of two players. Simplifying characterizations of the weak and strong sequential core are provided. Examples highlight the essential difference between these core concepts.
\end{abstract}

Keywords: cooperative games, dynamic games, core.

JEL classification: C71, C73.

*Department of Economics, University at Albany, SUNY, Albany, NY 12222. E-mail: L.Kranich@albany.edu.

†Departamento de Economía, Universidad Carlos III de Madrid. E-mail: Perea@eco.uc3m.es.

$\ddagger$ Department of Quantitative Economics, University of Maastricht, P.O. Box 616, 6200 MD Maastricht, The Netherlands. E-mail: H.Peters@ke.unimaas.nl. 


\section{Introduction}

The canonical representation of a cooperative game, the characteristic function, describes the payoffs available to each coalition of players. The simplest and most common interpretation of such a game is that it pertains to a single interaction among the players. However, many, if not most, cooperative endeavors occur more than once or even repeatedly over time.

A few papers have begun to lay the foundation for a theory of cooperative games played in dynamic settings, or dynamic cooperative games. The effort to develop a general theory of such games is complicated by the fact that various new issues arise in a dynamic context, and each may require a somewhat different treatment. In particular, whereas a standard, static, game specifies the set of players and the characteristic function, in a dynamic setting the population of players might change over time, or, given the set of players, the characteristic function might change either as a result of exogenous or endogenous forces. ${ }^{1}$ Typically, a solution to a dynamic game will exploit intertemporal linkages rather than simply apply a standard solution at each point in time. Moreover, the game itself may depend on the solution at each stage.

Rather than attempt to develop a single, overarching framework that would encompass all possibilities, the strategy adopted in this paper is to present a particular class of dynamic cooperative games and to focus on the extension of a particular solution concept, namely, the core. However, in contrast to static games it is not obvious how to define the core in a dynamic setting. Specifically, there are various ways in which one might formulate the notion of a "profitable deviation." Here we explore three different interpretations, each of which gives rise to a different notion of the core. After introducing the alternative core concepts, we investigate conditions ensuring their nonemptiness, and we consider the relationship between the various concepts.

Before discussing the core concepts, we briefly describe some of the key elements of a dynamic cooperative game. First, we will abstract from changes in the player set. Instead, our focus will be on finite horizon games in which at

\footnotetext{
${ }^{1}$ Rosenthal (1990a,1990b) considered the possibility of changing the player set over time, while keeping the worths of the original coalitions fixed. His study focuses on the monotonicity of solutions, that is, whether all of the original players gain with the inclusion of additional players.
} 
every stage the same players face an exogenously specified cooperative game with transferable utility. Typically, a solution to the dynamic model will specify a stream of payoffs for each player. This might involve exactly solving each stage game or, if it is possible to trade or transfer payoffs across periods, then it may be possible to relax the instantaneous resource constraints. In either case, it is essential that players have the means to evaluate payoffs at different points in time. Hence, included in the specification of a dynamic game will be the players' intertemporal preferences or utility.

Another important aspect of dynamic games is that the (stage) game and/or solution at any point in time might depend on the history of play up to that point, that is, they may be subject to endogenous influences. This was the subject of a recent paper by Filar and Petrosjan (2000), who considered dynamic cooperative games where each stage game may depend on the payoffs obtained in earlier periods. The focus of their study was on the issue of "time consistency" of solutions to the stage games. ${ }^{2}$ However, as mentioned above, here we abstract from endogenous influences and take the sequence of stage games to be given.

In addition to the papers cited above, various authors have addressed these or related issues often in the context of economic examples. For instance, Gale (1978), Becker and Chakrabarty (1995), and Koutsogeras (1998) considered core concepts for certain intertemporal economies. Predtechenski et al. (2001) considered both time and uncertainty in their study of the core of two-period economies with incomplete information. Finally, Munro (1979) considered Nash bargaining and the core in a dynamic model of optimal resource management, and Shalev (1995) formulated a repeated Nash bargaining model to explain loss aversion.

Turning back to the definition of the core, intuitively, in this context the core should capture those situations in which at each stage the grand coalition is formed, its worth is distributed among the players and no coalition has a profitable deviation. But in determining a profitable deviation, should coalitions be required to deviate at the start of the game and for all time, or can they deviate at a later stage? Should such a deviation itself be stable or are unstable deviations also to be considered? The three concepts we discuss provide different answers to these questions.

The first concept, termed the classical core, assumes that coalitions plan-

\footnotetext{
${ }^{2}$ Strotz (1955).
} 
ning to split off, do so from the beginning. It is not possible, therefore, for coalitions to separate from the grand coalition after having cooperated during the first stages. The classical core may thus be viewed as a static concept since it does not really depend on the time structure in the game. This is reflected by the fact that the classical core coincides with the core of an induced static cooperative game with nontransferable utility.

In the strong sequential core coalitions are allowed to deviate at any stage of the game, but done so in one period, they must do so in all remaining stages as well. The papers by Gale (1978) and Becker and Chakrabarti (1995), mentioned above, present similar core concepts for the special case of a monetary economy and a capital accumulation model, respectively. Also Koutsougeras (1998) presents a core concept in this spirit in a two-period economy with asset markets and differential information. For two-period economies with uncertainty and possibly asset markets, Predtechenski et al. (2001) study the strong sequential core.

In both the classical and strong sequential cores, deviations are not required to be stable. In other words, a coalition can deviate from the grand coalition and propose a distribution of its worth at each stage which, in turn, could be blocked by some subcoalition in the future. It is known that for static cooperative games this difference is inessential (Ray, 1989), since allowing only "credible" deviations (i.e. deviations which cannot be blocked by any subcoalition) does not enlarge the set of core allocations. In a dynamic setup, however, the focus on credible deviations does have an impact on the core concept. Payoff streams which distribute the worth of the grand coalition at every stage are said to be in the weak sequential core if they are robust against all credible deviations. Clearly, the strong sequential core is contained in both the classical core and the weak sequential core. It is shown by an example that there is no such logical relationship between the classical and weak sequential core: both cores may be nonempty but have an empty intersection.

With these different core concepts in hand, we then turn to two important issues. Can we find conditions on the stage games and the players' preferences which guarantee a nonempty core? And, is there an easy way to characterize core allocations, at least in some special cases? The last question is especially relevant for the weak sequential core, since its recursive definition of credible deviations makes it somewhat hard to handle.

The outline of the paper is as follows. In Section 2 we provide basic defi- 
nitions. Section 3 introduces the classical core concept and provides sufficient conditions on the stage games and preferences to ensure its nonemptiness. In brief, balancedness of the stage games together with quasiconcavity of preferences guarantee that the classical core is nonempty. An example shows that this condition is not necessary. In Section 4 we first describe the strong and weak sequential core concepts; we provide a characterization of weak sequential core allocations - which is used to prove an existence result for the two-player case - and we illustrate the concepts by examples. In the last part of this section, we investigate the different core concepts for two special preference classes: symmetric linear preferences and lexicographic preferences. Section 5 contains brief concluding remarks.

\section{Preliminaries}

In this section we provide preliminary definitions as well as some well known facts about the core. In addition we define the notion of a dynamic transferable utility game.

\section{$2.1 \quad$ TU-games}

A game with transferable utility or briefly a $T U$-game is a pair $(N, v)$, where $N:=\{1,2, \ldots, n\}$ is the set of players and $v: 2^{N} \rightarrow \mathbb{R}_{+}$assigns to each coalition $S \subseteq N^{3}$ its nonnegative worth $v(S)$, with the convention that $v(\emptyset)=$ 0 , and such that $v(S)>0$ for at least one coalition $S$. Usually we simply write $v$ instead of $(N, v)$. The central question in a TU-game is how to distribute the worth $v(S)$ among the members of $S$ if the coalition is formed. Such a distribution is also called a (payoff) allocation. For convenience we consider only nonnegative games, but allocations, in general, may contain negative components. The core of a TU-game $v$ is the set

$$
C(v):=\left\{x \in \mathbb{R}^{N}: x(N)=v(N) \text { and } x(S) \geq v(S) \text { for all } S \in 2^{N} \backslash\{\emptyset\}\right\},
$$

where $x(S)$ denotes the sum $\sum_{i \in S} x_{i}$.

A collection of coalitions $\mathcal{B} \subseteq 2^{N} \backslash\{\emptyset\}$ is balanced if there are positive real numbers $\lambda_{S}$ for every $S \in \mathcal{B}$ such that $\sum_{S \in \mathcal{B}: i \in S} \lambda_{S}=1$ for every $i \in N$. A

\footnotetext{
${ }^{3}$ The symbol $\subseteq$ means 'inclusion' and the symbol $\subset$ means 'strict inclusion'. Vector inequalities are denoted $\geq,>$, and $\leq,<$.
} 
TU-game $v$ is balanced if $\sum_{S \in \mathcal{B}} \lambda_{S} v(S) \leq v(N)$ for every balanced collection $\mathcal{B}$. According to the well known result of Bondareva (1963) and Shapley (1967), a TU-game has a nonempty core if and only if it is balanced.

\section{$2.2 \quad$ NTU-games}

A game without transferable utility or briefly an $N T U$-game is a pair $(N, V)$ where $V(S) \subseteq \mathbb{R}^{N}$ for each coalition $S$, and $V(\emptyset)=\emptyset$. Usually we write $V$ instead of $(N, V)$. Often the following additional conditions are imposed on an NTU-game $V$ :

(N.1) for all $S \neq \emptyset, V(S)$ is nonempty and closed,

(N.2) if $x \in V(S)$ and $y_{i} \leq x_{i}$ for all $i \in S$, then $y \in V(S)$,

(N.3) for every $i \in N$ there is an $m_{i} \in \mathbb{R}$ with $V(\{i\})=\left\{x \in \mathbb{R}^{N}: x_{i} \leq m_{i}\right\}$, and $V(N) \cap\left\{x \in \mathbb{R}^{N}: x_{i} \geq m_{i}\right.$ for all $\left.i \in N\right\}$ is compact.

The core of an NTU-game $V$, denoted by $C(V)$, is the set $V(N) \backslash \cup_{S \subseteq N} \operatorname{int} V(S)$. (Here 'int' denotes 'interior'.) An NTU-game $V$ is balanced if $\cap_{S \in \mathcal{B}} V(S) \subseteq$ $V(N)$ for every balanced collection $\mathcal{B}$. Scarf (1967) proved that if $V$ satisfies (N.1)-(N.3) and is balanced then it has a nonempty core (see also Kannai, 1992).

\subsection{Dynamic TU-games}

Let $T$ be a natural number and let $\mathbf{v}=\left(v^{1}, v^{2}, \ldots, v^{T}\right)$ be a sequence of TU-games with the same set of players. Let $u=\left(u_{i}\right)_{i \in N}$ be a profile of utility functions for the players. Here, $u_{i}: \mathbb{R}^{T} \rightarrow \mathbb{R}$ is a function assigning to every payoff stream $\mathbf{x}_{i}=\left(x_{i}^{1}, \ldots, x_{i}^{T}\right)$ for player $i$ some utility $u_{i}\left(\mathbf{x}_{i}\right)$. Unless explicitly stated otherwise, we assume that the utility functions $u_{i}$ are continuous and strictly increasing in each coordinate, and that each $u_{i}$ is time separable (Koopmans, 1960). The last assumption means that for every stage $t$, every pair $\left(x^{1}, \ldots, x^{t-1}\right),\left(\tilde{x}^{1}, \ldots, \tilde{x}^{t-1}\right)$ and every pair $\left(y^{t}, \ldots, y^{T}\right)$, $\left(\tilde{y}^{t}, \ldots, \tilde{y}^{T}\right)$ we have:

$$
u_{i}\left(x^{1}, \ldots, x^{t-1}, y^{t}, \ldots, y^{T}\right) \geq u_{i}\left(x^{1}, \ldots, x^{t-1}, \tilde{y}^{t}, \ldots, \tilde{y}^{T}\right)
$$


if and only if

$$
u_{i}\left(\tilde{x}^{1}, \ldots, \tilde{x}^{t-1}, y^{t}, \ldots, y^{T}\right) \geq u_{i}\left(\tilde{x}^{1}, \ldots, \tilde{x}^{t-1}, \tilde{y}^{t}, \ldots, \tilde{y}^{T}\right) .
$$

In words, the evaluation of future allocation streams does not depend on the history up to that point. Time separability will enable us to define utility functions from any moment $t=1, \ldots, T$ on, independent of the preceding allocation stream (see Section 4.1).

Not all of these assumptions are needed for every result in the sequel. The assumption of time separability will be needed when we discuss the weak and strong sequential cores in Section 4.

One simple way to interpret the utility function $u_{i}$ is to think of the payoffs in a static TU-game as money: then $u_{i}$ represents the evaluation of streams of money. Alternative interpretations are possible, depending on the situation. E.g., utility might be additive-possibly with discounting-over time.

The pair $\Gamma=(\mathbf{v}, u)$ is called a dynamic TU-game. A feasible allocation stream for a coalition $S$ in $\Gamma$ is a sequence $\mathbf{y}_{S}=\left(y^{1}, \ldots, y^{T}\right) \in \mathbb{R}^{S \times T}$ such that $y^{t}(S)=v^{t}(S)$ for every $t=1, \ldots, T$. (Note that bold-face symbols denote time indexed vectors.) For $S=N$, we omit the ' $N$ ' and write $\mathbf{y}$ rather than $\mathbf{y}_{N}$. Let $Z_{\Gamma}(S)$ denote the set of feasible allocation streams for $S$ in $\Gamma$. Also, for $\mathbf{y}_{S} \in Z_{\Gamma}(S)$, we write $u\left(\mathbf{y}_{S}\right)$ in place of $\left(u_{i}\left(\mathbf{y}_{i}\right)\right)_{i \in S}$.

For a dynamic TU-game $\Gamma=(\mathbf{v}, u)$ and a nonempty coalition $S$, we define the set $V_{\Gamma}(S)$ by

$$
V_{\Gamma}(S):=\left\{x \in \mathbb{R}^{N}: \text { for some } \mathbf{y}_{S} \in Z_{\Gamma}(S), x \leq u\left(\mathbf{y}_{S}\right)\right\} .
$$

With $V_{\Gamma}(\emptyset):=\emptyset$, the pair $\left(N, V_{\Gamma}\right)$ is an NTU-game satisfying conditions (N.1), (N.2) and (N.3). We refer to $V_{\Gamma}$ as the NTU-game associated with $\Gamma$. $V_{\Gamma}$ represents the situation where coalitions cooperate in every stage of the game.

\section{The classical core}

The classical core of a dynamic TU-game $\Gamma=(\mathbf{v}, u)$, denoted by $C(\Gamma)$ or $C(\mathbf{v}, u)$, consists of those allocation streams that generate utility profiles in the core of the associated NTU-game $V_{\Gamma}$, that is,

$$
C(\Gamma):=\left\{\mathbf{y}=\left(y^{1}, \ldots, y^{T}\right) \in \mathbb{R}^{N \times T}: \mathbf{y} \in Z_{\Gamma}(N) \text { and } u(\mathbf{y}) \in C\left(V_{\Gamma}\right)\right\} .
$$


In this section we investigate conditions under which the classical core is non-empty. Here, the specific time structure does not play a role, and all results would obviously hold without the assumption of time separability. ${ }^{4}$

In our first result we establish directly that the classical core is nonempty provided the core of each stage game is nonempty as well. The proof associates a dynamic TU-game with an intertemporal exchange economy in which the worth of the grand coalition must be allocated in each period. Nonemptiness of the classical core follows from the existence of a competitive equilibrium in such a context. It is interesting to note that, whereas the core of an exchange economy results from an application of game theory to economics, here we solve a game theoretic problem by employing tools from economics.

Theorem 1 Let $\Gamma=(\mathbf{v}, u)$ be a dynamic TU-game satisfying:

(i) $C\left(v^{t}\right) \neq \emptyset$ for every $t=1, \ldots, T$,

(ii) $u_{i}$ is quasiconcave for every $i \in N$.

Then $C(\Gamma) \neq \emptyset$.

Proof. First, we construct an artificial "exchange economy" in which the vector $\mathbf{v}(N)=\left(v^{1}(N), \ldots, v^{T}(N)\right)$ is to be allocated among the $n$ agents, and the preferences of the agents are represented by their respective utility functions $u_{i}\left(\mathbf{y}_{i}\right)$. We then identify a suitable "initial allocation," and show that a "competitive equilibrium" from the initial allocation is in $C(\Gamma)$. Specifically, we claim that any $\mathbf{y} \in C\left(v^{1}\right) \times \cdots \times C\left(v^{T}\right) \subset \mathbb{R}^{N \times T}$ would serve as a suitable initial allocation.

Let $\mathbf{y}^{\circ}$ denote such an allocation. Under the assumptions on $u_{i}$, a competitive equilibrium from $\mathbf{y}^{\circ}$ exists: recall that the utility functions are strictly increasing, and since by assumption $v^{t}(S)>0$ for some $S \subseteq N$ and $C\left(v^{t}\right) \neq \emptyset$, $v^{t}(N)>0$. Let $\mathbf{y}^{*} \in \mathbb{R}^{N \times T}$ denote an equilibrium allocation and $\mathbf{p}^{*}$ the supporting prices. Note that $\mathbf{p}^{*}>0$. We need only show that $\mathbf{y}^{*} \in C(\Gamma)$.

By way of contradiction, suppose $\mathbf{y}^{*} \notin C(\Gamma)$. Then there exists $S \subseteq N$ and some $\mathbf{y}_{S} \in Z_{\Gamma}(S)$ such that $u_{i}\left(\mathbf{y}_{i}\right)>u_{i}\left(\mathbf{y}_{i}^{*}\right)$ for all $i \in S$, and $\sum_{i \in S} \mathbf{y}_{i}=$

\footnotetext{
${ }^{4}$ If the specific time structure does not play a role, then an alternative formal interpretation of the periods $1, \ldots, T$ is as states of nature. With this interpretation, the classical core corresponds to the ex ante core, i.e., before the uncertainty is resolved.
} 
$\mathbf{v}(S)$. Since the utility functions are strictly increasing, it must be the case that for all $i \in S, \mathbf{p}^{*} \cdot \mathbf{y}_{i}>\mathbf{p}^{*} \cdot \mathbf{y}_{i}^{*}$. Hence, $\mathbf{p}^{*} \cdot \sum_{i \in S} \mathbf{y}_{i}=\mathbf{p}^{*} \cdot \mathbf{v}(S)>\mathbf{p}^{*} \cdot \sum_{i \in S} \mathbf{y}_{i}^{*}$. However, since $\mathbf{y}^{*}$ is an equilibrium allocation, $\mathbf{p}^{*} \cdot \mathbf{y}_{i}^{*}=\mathbf{p}^{*} \cdot \mathbf{y}_{i}^{\circ}$ for all $i \in S$. Summing over $S, \mathbf{p}^{*} \cdot \sum_{i \in S} \mathbf{y}_{i}^{*}=\mathbf{p}^{*} \cdot \sum_{i \in S} \mathbf{y}_{i}^{\circ}$. Since $\mathbf{y}^{\circ} \in C\left(v^{1}\right) \times \cdots \times C\left(v^{T}\right)$, $\sum_{i \in S} \mathbf{y}_{i}^{\circ} \geq \mathbf{v}(S)$. Hence, $\mathbf{p}^{*} \cdot \sum_{i \in S} \mathbf{y}_{i}^{*} \geq \mathbf{p}^{*} \cdot \mathbf{v}(S)$, which is a contradiction.

Our second existence result is slightly more general. We establish that under the same conditions as in Theorem 1 the NTU-game associated with $\Gamma$ is balanced. Nonemptiness of the core then follows by the result of Scarf (1967) since this NTU-game satisfies conditions (N1)-(N3).

Theorem 2 Let $\Gamma=(\mathbf{v}, u)$ be a dynamic TU-game satisfying:

(i) $C\left(v^{t}\right) \neq \emptyset$ for every $t=1, \ldots, T$,

(ii) $u_{i}$ is quasiconcave for every $i \in N$.

Then $V_{\Gamma}$ is balanced.

Proof. Let $\mathcal{B}$ be a balanced collection with weights $\lambda_{S}$ for every $S \in \mathcal{B}$. Let $x \in \bigcap_{S \in \mathcal{B}} V_{\Gamma}(S)$. It is sufficient to show that $x \in V_{\Gamma}(N)$. For every $S \in \mathcal{B}$ take vectors $\mathbf{y}_{S}=\left(y^{1, S}, \ldots, y^{T, S}\right)(t=1, \ldots, T)$ as in the definition of $V_{\Gamma}(S)$ in Section 2. Define $\mathbf{Y} \in \mathbb{R}^{N \times T}$ by

$$
\mathbf{Y}_{i}:=\sum_{S \in \mathcal{B}: i \in S} \lambda_{S}\left(y_{i}^{1, S}, \ldots, y_{i}^{T, S}\right)
$$

for every $i \in N$. By the quasiconcavity of $u_{i}$, we have $x_{i} \leq u_{i}\left(\mathbf{Y}_{i}\right)$ for all $i$. Hence, it suffices to show that $\mathbf{Y}=\left(\mathbf{Y}_{1}, \ldots, \mathbf{Y}_{n}\right)$ is feasible for the grand coalition. Now

$$
\begin{aligned}
\sum_{i \in N} \mathbf{Y}_{i} & =\left(\sum_{S \in \mathcal{B}} \lambda_{S} \sum_{i \in S} y_{i}^{1, S}, \ldots, \sum_{S \in \mathcal{B}} \lambda_{S} \sum_{i \in S} y_{i}^{T, S}\right) \\
& =\left(\sum_{S \in \mathcal{B}} \lambda_{S} v^{1}(S), \ldots, \sum_{S \in \mathcal{B}} \lambda_{S} v^{T}(S)\right) \\
& \leq\left(v^{1}(N), \ldots, v^{T}(N)\right)
\end{aligned}
$$


where the inequality follows from balancedness (Bondareva-Shapley) of the TU-games $v^{t}$. This shows that $\mathbf{Y}$ is feasible for the grand coalition and, hence, $x \in V_{\Gamma}(N)$.

The following example illustrates that, while it is sufficient that the stage games have nonempty cores, this is by no means necessary for the dynamic game to have a nonempty core.

Example 1 Let $T=2, N=\{1,2\}, v^{1}(\{1\})=v^{2}(\{2\})=2, v^{1}(\{2\})=$ $v^{2}(\{1\})=5$, and $v^{1}(\{1,2\})=v^{2}(\{1,2\})=6.5$. Then $C\left(v^{1}\right)=C\left(v^{2}\right)=\emptyset$. Let $u_{i}\left(x_{i}^{1}, x_{i}^{2}\right)=x_{i}^{1} x_{i}^{2}$ for $i=1,2$, and $\Gamma=(\mathbf{v}, u) .{ }^{5}$ Then $C(\Gamma) \neq \emptyset$ and it is given by

$$
\begin{aligned}
C(\Gamma)= & \left\{\left(\left(x_{1}^{1}, x_{1}^{2}\right),\left(x_{2}^{1}, x_{2}^{2}\right)\right): x_{1}^{1}=x_{1}^{2}, x_{2}^{1}=x_{2}^{2}=6.5-x_{1}^{1},\right. \\
& \left.\sqrt{10} \leq x_{1}^{1} \leq 6.5-\sqrt{10}\right\} .
\end{aligned}
$$

The fact that in Example 1 and in many other examples (see below) the classical core of the dynamic game is nonempty in spite of the fact that the stage TU-games have empty cores is caused by the possibility of implicit utility transfers between periods. In order to capture this idea we will present another existence result on the classical core in which these utility transfers are formalized in a specific sense.

Let $\Gamma=(\mathbf{v}, u)$ be a dynamic TU-game. For every nonempty coalition $S$ and every $\alpha \in \mathbb{R}$ define

$$
\begin{aligned}
V_{u}^{\alpha}(S):= & \left\{x \in \mathbb{R}^{N}: \text { for every } i \in S \text { there is a } \mathbf{y}_{i}=\left(y_{i}^{1}, \ldots, y_{i}^{T}\right)\right. \\
& \in \mathbb{R}^{T} \text { such that } \sum_{i \in S} \sum_{t=1}^{T} y_{i}^{t}=\alpha \text { and } x_{i} \leq u_{i}\left(\mathbf{y}_{i}\right) \\
& \text { for every } i \in S\} .
\end{aligned}
$$

Note that $V_{u}^{\alpha}(S)$ does not depend on $\mathbf{v}$, but it does depend on $u$. It consists of the set of those utility allocations that can be obtained by distributing the amount $\alpha$ among the players in $S$. Let $\alpha(\mathbf{v}(S))$ be the minimal value of

\footnotetext{
${ }^{5}$ These utility functions satisfy all of our assumptions only for positive allocations, which are the only ones that are relevant in this example. Alternatively, one may take for instance utility functions $u_{i}\left(x_{i}^{1}, x_{i}^{2}\right)=-e^{-x_{i}^{1}}-e^{-x_{i}^{2}}$, which satisfy all of our assumptions for all possible allocations but are slightly less easy to work with.
} 
$\alpha$ for which $V_{u}^{\alpha}(S) \supseteq V_{\Gamma}(S)$. Note that $\alpha(\mathbf{v}(S)) \leq \sum_{t=1}^{T} v^{t}(S)$. For possible reference we state the following observation as a theorem. In this theorem we define an NTU-game $\tilde{V}$ such that for the grand coalition the individually rational part of the feasible set is contained in that of $V_{\Gamma}$, and for each smaller coalition the feasible set contains that of $V_{\Gamma}$. This is done in a tight way by distributing fixed amounts among the coalitions. These fixed amounts are at most equal to what each coalition could generate if it had the possibility to pool its worth over all time periods. Thus, it tries to capture the idea of transfers between periods.

Theorem 3 Let $\Gamma=(\mathbf{v}, u)$ be a dynamic TU-game. Define the NTU-game $\tilde{V}$ by $\tilde{V}(S):=V_{u}^{\alpha(\mathbf{v}(S))}(S)$ for $S \neq N$ and $\tilde{V}(N):=V_{u}^{\tilde{\alpha}}(N)$, where

$$
\begin{aligned}
\tilde{\alpha}:= & \max \left\{\alpha: \forall x \in \mathbb{R}^{N}\left[x \in V _ { u } ^ { \alpha } ( N ) \text { and } x \notin \operatorname { i n t } \left(V_{\Gamma}(\{i\}) \forall i \in N\right.\right.\right. \\
& \left.\left.\Rightarrow x \in V_{\Gamma}(N)\right]\right\} .
\end{aligned}
$$

Then $C(\tilde{V}) \neq \emptyset$ implies $C(\Gamma) \neq \emptyset$.

Proof. By definition, $\tilde{V}(S)=V_{u}^{\alpha(\mathbf{v}(S))}(S) \supseteq V_{\Gamma}(S)$ for $S \neq N$, while $\tilde{V}(N) \cap$ $\left\{x \in \mathbb{R}^{N}: \forall i \in N\left[x \notin \operatorname{int} V_{\Gamma}(\{i\})\right]\right\} \subseteq V_{\Gamma}(N)$. From this the theorem follows.

For this result to be useful one has to know when the NTU-game $\tilde{V}$ has a nonempty core. More generally, let $\Gamma^{\prime}=(w, u)$ be a 'trivial' dynamic game, i.e., $T=1$. Then the assumption of strictly increasing utility functions implies $C\left(\Gamma^{\prime}\right)=C(w)$.

Example 2 Consider again the game in Example 1. In this case $\alpha(\mathbf{v}(\{1\}))=\alpha(\mathbf{v}(\{2\}))=2 \sqrt{10}, \tilde{\alpha}=13$. The resulting game $\tilde{V}$ is the game $V_{\Gamma^{\prime}}$ where $\Gamma^{\prime}=(w, u)$ with $w(\{1\})=w(\{2\})=2 \sqrt{10}$ and $w(\{1,2\})=13$. Thus, $C\left(\Gamma^{\prime}\right)=C(w) \neq \emptyset$, and therefore by Theorem $3 C(\Gamma) \neq \emptyset$.

Example 3 Consider the two-period three-player game $\Gamma=(\mathbf{v}, u)$ defined by $v^{1}(\{1,2\})=4, v^{1}(\{2,3\})=2, v^{2}(\{1,2\})=2, v^{2}(\{2,3\})=4, v^{1}(N)=$ $v^{2}(N)=2.9$, and $v^{1}(S)=v^{2}(S)=0$ otherwise; and $u_{i}\left(x_{i}^{1}, x_{i}^{2}\right)=x_{i}^{1} x_{i}^{2}$ for $i=1,2,3$ (cf. footnote 5). Then $v^{1}$ and $v^{2}$ are not balanced (and neither is $\left.v^{1}+v^{2}\right)$. In this case $\alpha(\mathbf{v}(S))=2 \sqrt{8}$ for $S=\{1,2\}$ and $S=\{2,3\}$, and $\tilde{\alpha}=5.8$. The game $\tilde{V}$ is derived from the TU-game $w$ with $w(\{1,2\})=$ 
$w(\{2,3\})=2 \sqrt{8}, w(N)=5.8$, and $v(S)=0$ otherwise. The game $w$ is balanced, hence $(w, u)$ has a nonempty core, and by Theorem 3 the dynamic game $\Gamma=(\mathbf{v}, u)$ has a nonempty classical core.

\section{The weak and the strong sequential core}

First, definitions of core concepts will be proposed that capture the time structure of a dynamic game. An alternative description of one of these, the weak sequential core, is provided by Lemma 1. Theorem 4 provides an existence result for the weak sequential core in the case of two players. In Section 4.2 relationships between the three core concepts or the absence thereof are illustrated by examples. The last two sections are devoted to special cases where preferences are linear and symmetric, or lexicographic, respectively.

\subsection{Definitions}

Since every utility function $u_{i}$ is assumed to be time separable, there is a utility function $u_{i}^{t}: \mathbb{R}^{T-t+1} \rightarrow \mathbb{R}$ for each stage $t$ such that for every $\left(x^{1}, \ldots, x^{t-1}\right)$ and every pair $\left(y^{t}, \ldots, y^{T}\right),\left(\tilde{y}^{t}, \ldots, \tilde{y}^{T}\right)$ we have:

$$
u_{i}\left(x^{1}, \ldots, x^{t-1}, y^{t}, \ldots, y^{T}\right) \geq u_{i}\left(x^{1}, \ldots, x^{t-1}, \tilde{y}^{t}, \ldots, \tilde{y}^{T}\right)
$$

if and only if

$$
u_{i}^{t}\left(y^{t}, \ldots, y^{T}\right) \geq u_{i}^{t}\left(\tilde{y}^{t}, \ldots, \tilde{y}^{T}\right) .
$$

It is therefore possible to define the core of subgames. The subgame starting at stage $t$ is the dynamic cooperative game $\Gamma^{t}=\mathbf{v}^{t}:=\left(\left(v^{t}, \ldots, v^{T}\right),\left(u_{i}^{t}\right)_{i \in N}\right)$. The core of the subgame $\Gamma^{t}$ is defined as the classical core of the dynamic cooperative game $\Gamma^{t}$.

Let $\Gamma=(\mathbf{v}, u)$ be a dynamic TU-game and let $\mathbf{x}$ be an allocation stream. By $\mathbf{x}^{t}$ we denote the allocation stream from time $t$ on, i.e., $\mathbf{x}^{t}=\left(x^{t}, \ldots, x^{T}\right)$. More generally, $\mathbf{x}^{t}$ may denote an allocation stream from time $t$ on.

Definition 1 A deviation by $S \subseteq N$ at time $t$ from $\mathbf{x}$ is a feasible allocation stream $\mathbf{y}^{t}=\left(y^{t}, \ldots, y^{T}\right) \in\left(\mathbb{R}^{S}\right)^{T-t+1}$ for the coalition $S$ such that $u_{i}^{t}\left(\mathbf{y}_{i}^{t}\right)>$ $u_{i}^{t}\left(\mathbf{x}_{i}^{t}\right)$ for all $i \in S$. 
Note that by defining a deviation in this way we implicitly assume that a coalition, once it deviates, it deviates for the rest of the time.

Definition 2 The strong sequential core of $\Gamma=(\mathbf{v}, u)$ is the set of feasible allocation streams $\mathbf{x}$ for the grand coalition from which no coalition ever has a deviation.

Remark 1 Note that $\mathbf{x}$ is in the strong sequential core of $\Gamma=(\mathbf{v}, u)$ if, and only if, at every stage $t$, the continuation stream $\mathbf{x}^{t}=\left(x^{t}, \ldots, x^{T}\right)$ is in the (classical) core of the subgame $\Gamma^{t}=\left(\left(v^{t}, \ldots, v^{T}\right),\left(u_{i}^{t}\right)_{i \in N}\right)$. Therefore, the existence of a strong sequential core allocation implies the nonemptiness of all these classical cores.

We denote the strong sequential core by $S S C(\Gamma)$. In its definition, we allow coalitions $S$ to deviate by allocation streams $\mathbf{y}^{t}$ which, themselves, can be improved upon in the future by subcoalitions of $S$. The following definitions lead to a weakening of this core concept by putting an additional requirement on deviations.

Definition 3 A deviation $\mathbf{y}^{t}$ as in Definition 1 is credible if there is no $S^{\prime} \subseteq S$ and a time $t^{\prime} \geq t$ such that $S^{\prime}$ has a deviation at $t^{\prime}$ from $\mathbf{y}^{t}$, i.e., a feasible allocation stream $\left(y^{\prime t^{\prime}}, \ldots, y^{\prime T}\right)$ for $S^{\prime}$ with $u_{i}^{t^{\prime}}\left(\mathbf{y}_{i}^{\prime t^{\prime}}\right)>u_{i}^{t^{\prime}}\left(\mathbf{y}_{i}^{t^{\prime}}\right)$ for all $i \in S^{\prime}$.

Definition 4 The weak sequential core of $\Gamma=(\mathbf{v}, u)$ is the set of feasible allocation streams $\mathbf{x}$ for the grand coalition from which no coalition ever has a credible deviation.

We denote the weak sequential core by $W S C(\Gamma)$. Obviously, the strong sequential core is always a subset of the weak sequential core. One might argue that the definition of the weak sequential core is too restrictive, since it would be natural to require a deviation by a coalition $S^{\prime}$ as in Definition 3 to be credible at its turn. It is easy to show, however, that existence of a deviation by $S^{\prime}$ implies the existence of a credible deviation. For suppose that $S^{\prime}$ at $t^{\prime}$ has a deviation $\mathbf{y}^{\prime t^{\prime}}$ from $\mathbf{y}^{t}$ that is not credible. We may assume that $S^{\prime}$ itself does not have a deviation from this deviation (otherwise continue with a deviation from which $S^{\prime}$ itself does not have a deviation). Then there is a coalition $S^{\prime \prime} \subset S^{\prime}$ that has at time $t^{\prime \prime} \geq t^{\prime}$ a deviation $\mathbf{y}^{\prime \prime t^{\prime \prime}}$ from $\mathbf{y}^{\prime t^{\prime}}$. If this deviation is credible then it is obviously also a credible deviation 
from $\mathbf{x}$. Otherwise we can repeat the argument, possibly until we end up at one-person coalitions, which always have a credible deviation if they have a deviation. Thus, for the weak sequential core it does not make a difference if we would add this additional requirement. ${ }^{6}$

The weak sequential core can be characterized in a simple way. For a TU-game $(N, v)$ and a coalition $S \subseteq N$ the restriction of $v$ to $S$ is denoted by $(S, v)$. For a dynamic game $\mathbf{v}$ the notation $(S, \mathbf{v})$ similarly denotes the restriction of the game to $S$ at every moment $t$.

Lemma $\mathbf{1}$ Let $\mathbf{v}$ be a dynamic game, and $\mathbf{x}$ a feasible allocation stream for $N$. Then the following two statements are equivalent.

(a) $\mathbf{x}=\left(x^{1}, x^{2}, \ldots, x^{T}\right) \in W S C(\mathbf{v})$,

(b) $\mathbf{x}^{2}=\left(x^{2}, \ldots, x^{T}\right) \in W S C\left(\mathbf{v}^{2}\right)$ and there are no $S \subseteq N$ and allocation stream $\left(y^{1}, y^{2}, \ldots, y^{T}\right)$ such that $y^{1}(S)=v^{1}(S),\left(y^{2}, \ldots, y^{T}\right) \in$ $W S C\left(S, \mathbf{v}^{2}\right)$ and $u_{i}\left(\mathbf{y}_{i}\right)>u_{i}\left(\mathbf{x}_{i}\right)$ for all $i \in S$.

Proof. For the implication $(\mathrm{a}) \Rightarrow(\mathrm{b})$ we only still have to show that if an allocation stream $\left(y^{1}, y^{2}, \ldots, y^{T}\right)$ as in (b) would exist, then there would also exist a credible deviation, thus contradicting (a). But this is obvious because if $\left(y^{1}, y^{2}, \ldots, y^{T}\right)$ is itself not credible, then we can always find another credible deviation at $t=1$, by an argument analogous to the one following Definition 4. For the implication $(\mathrm{b}) \Rightarrow(\mathrm{a})$ we similarly only have to show that there is no credible deviation at $t=1$, but this is immediate.

Note that this lemma clarifies the essentially recursive nature of the weak sequential core. This will be seen again in the examples that follow below. First we provide an existence result for the weak sequential core in the case of two players. For this result we use the following observation.

Lemma 2 Let $(\mathbf{v}, u)$ be a dynamic cooperative game and for every $t \in$ $\{1, \ldots, T\}$ let $u_{i}^{t}$ be the induced utility function for allocation streams in the subgame $\mathbf{v}^{t}$. Then for all $\mathbf{x}^{t}=\left(x^{t}, \mathbf{x}^{t+1}\right)$ and $\mathbf{y}^{t}=\left(y^{t}, \mathbf{y}^{t+1}\right)$ with with $x_{i}^{t} \leq y_{i}^{t}$ and $u_{i}^{t+1}\left(\mathbf{x}_{i}^{t+1}\right) \leq u_{i}^{t+1}\left(\mathbf{y}_{i}^{t+1}\right)$ it holds that $u_{i}^{t}\left(\mathbf{x}_{i}^{t}\right) \leq u_{i}^{t}\left(\mathbf{y}_{i}^{t}\right)$.

\footnotetext{
${ }^{6}$ This is analogous to equality of core and so-called modified core as shown in Ray (1989).
} 
Proof. Follows directly from the time separability and monotonicity of the utility functions.

Theorem 4 Let $\Gamma=(\mathbf{v}, u)$ be a dynamic cooperative game with two players and with $C\left(v^{t}\right) \neq \emptyset$ for every $t=1, \ldots, T$. Then $W S C(\Gamma) \neq \emptyset$.

Proof. For every $t \in\{1, \ldots, T\}$, let $I R\left(\mathbf{v}^{t}\right)$ be the set of those allocation streams $\mathbf{x}^{t}$ in $\mathbf{v}^{t}$ that are feasible for the grand coalition and for which $u_{i}^{t}\left(\mathbf{x}_{i}^{t}\right) \geq u_{i}^{t}\left(v^{t}(\{i\}), \ldots, v^{T}(\{i\})\right)$ for both players $i$. Let $X^{t}=\left\{\left(x_{1}^{t}, x_{2}^{t}\right) \in\right.$ $\left.\mathbb{R}^{2}: x_{1}^{t}+x_{2}^{t}=v^{t}(N)\right\}$ be the set of feasible allocations for the grand coalition at stage $t$. For every set $A$ of allocation streams in $\mathbf{v}^{t}$, let $P O(A)$ be the set of Pareto optimal allocation streams in $A$. By Lemma 1, it may be verified easily that

$$
\begin{aligned}
W S C\left(\mathbf{v}^{T}\right) & =C\left(v^{T}\right), \\
W S C\left(\mathbf{v}^{t}\right) & =I R\left(\mathbf{v}^{t}\right) \cap P O\left(X^{t} \times W S C\left(\mathbf{v}^{t+1}\right)\right)
\end{aligned}
$$

for all $t<T$. We show by induction on $t$ that $W S C\left(\mathbf{v}^{t}\right)$ is nonempty and compact for all $t$.

By assumption, $C\left(v^{T}\right)$ is nonempty. Since $C\left(v^{T}\right)$ is also compact, we have that $W S C\left(\mathbf{v}^{T}\right)$ is nonempty and compact.

Now, let $t<T$, and assume that $W S C\left(\mathbf{v}^{t+1}\right)$ is nonempty and compact. By continuity of the utility functions, we know that $I R\left(\mathbf{v}^{t}\right)$ and $P O\left(X^{t} \times W S C\left(\mathbf{v}^{t+1}\right)\right)$ are both compact, and hence it remains to show that $\operatorname{IR}\left(\mathbf{v}^{t}\right) \cap P O\left(X^{t} \times W S C\left(\mathbf{v}^{t+1}\right)\right)$ is nonempty. Let $\mathbf{y}^{t+1} \in W S C\left(\mathbf{v}^{t+1}\right)$ be arbitrary. Let $\mathbf{y}^{t}=\left(\left(v^{t}(\{1\}), v^{t}(N)-v^{t}(\{1\})\right), \mathbf{y}^{t+1}\right) \in X^{t} \times W S C\left(\mathbf{v}^{t+1}\right)$. Let $\mathbf{z}^{t} \in X^{t} \times W S C\left(\mathbf{v}^{t+1}\right)$ be such that $u_{1}^{t}\left(\mathbf{z}_{1}^{t}\right) \geq u_{1}^{t}\left(\mathbf{y}_{1}^{t}\right)$ and $u_{2}^{t}\left(\mathbf{z}_{2}^{t}\right)=$ $\max \left(\left\{u_{2}^{t}\left(\mathbf{x}_{2}^{t}\right): \mathbf{x}^{t} \in X^{t} \times W S C\left(\mathbf{v}^{t+1}\right)\right.\right.$ and $\left.u_{1}^{t}\left(\mathbf{x}_{1}^{t}\right) \geq u_{1}^{t}\left(\mathbf{y}_{1}^{t}\right)\right\}$. Then, by construction, $\mathbf{z}^{t} \in P O\left(X^{t} \times W S C\left(\mathbf{v}^{t+1}\right)\right)$. We prove that $\mathbf{z}^{t} \in I R\left(\mathbf{v}^{t}\right)$. By construction, $u_{1}^{t}\left(\mathbf{z}_{1}^{t}\right) \geq u_{1}^{t}\left(\mathbf{y}_{1}^{t}\right)$. By definition, $\mathbf{y}^{t}=\left(\left(v^{t}(\{1\}), v^{t}(N)-\right.\right.$ $\left.\left.v^{t}(\{1\})\right), \mathbf{y}^{t+1}\right)$ with $\mathbf{y}^{t+1} \in W S C\left(\mathbf{v}^{t+1}\right) \subseteq I R\left(\mathbf{v}^{t+1}\right)$ and hence $u_{1}^{t+1}\left(\mathbf{y}_{1}^{t+1}\right) \geq$ $u_{1}^{t+1}\left(\left(v^{t+1}(\{1\}), \ldots, v^{T}(\{1\})\right.\right.$.

By Lemma $2, u_{1}^{t}\left(\mathbf{z}_{1}^{t}\right) \geq u_{1}^{t}\left(\left(v^{t}(\{1\}), \ldots, v^{T}(\{1\})\right)\right.$.

On the other hand, $u_{2}^{t}\left(\mathbf{z}_{2}^{t}\right) \geq u_{2}^{t}\left(\mathbf{y}_{2}^{t}\right)$. By definition, $\mathbf{y}^{t}=\left(\left(v^{t}(\{1\}), v^{t}(N)-\right.\right.$ $\left.\left.v^{t}(\{1\})\right), \mathbf{y}^{t+1}\right)$. Since $C\left(v^{t}\right)$ is nonempty, it holds that $v^{t}(N)-v^{t}(\{1\}) \geq$ $v^{t}(\{2\})$. By construction, $\mathbf{y}^{t+1} \in W S C\left(\mathbf{v}^{t+1}\right) \subseteq I R\left(\mathbf{v}^{t+1}\right)$ and hence $u_{2}^{t+1}\left(\mathbf{y}_{2}^{t+1}\right) \geq u_{2}^{t+1}\left(\left(v^{t+1}(\{2\}), \ldots, v^{T}(\{2\})\right.\right.$. By Lemma 2 ,

it follows that $u_{2}^{t}\left(\mathbf{y}_{2}^{t}\right) \geq u_{2}^{t}\left(\left(v^{t}(\{2\}), \ldots, v^{T}(\{2\})\right.\right.$. We may thus conclude that 
$u_{2}^{t}\left(\mathbf{z}_{2}^{t}\right) \geq u_{2}^{t}\left(v^{t}(\{2\}), \ldots, v^{T}(\{2\})\right)$, and hence

$\mathbf{z}^{t} \in I R\left(\mathbf{v}^{t}\right) \cap P O\left(X^{t} \times W S C\left(\mathbf{v}^{t+1}\right)\right)=W S C\left(\mathbf{v}^{t}\right)$. This completes the proof.

Example 5 below shows that Theorem 4 does not hold for more than two players.

\subsection{Examples}

From the definition, it is clear that the strong sequential core is always contained in the classical core. Moreover, the strong sequential core is always a subset of the weak sequential core, since the former allows for a more general class of deviations and is therefore more restrictive. Between the weak sequential core and the classical core, there is no logical relationship. It is even possible for both sets to be non-empty but disjoint, as is illustrated by the following example.

Example 4 Let $N=\{1,2\}$ and $T=2$. Let the stage games be given by

$$
\begin{aligned}
& v^{1}(\{1\})=8, v^{1}(2)=2, v^{1}(\{1,2\})=10 \\
& v^{2}(\{1\})=2, v^{2}(2)=8, v^{2}(\{1,2\})=10 .
\end{aligned}
$$

Let the utility functions be

$$
u_{i}\left(x_{i}^{1}, x_{i}^{2}\right)=x_{i}^{1} x_{i}^{2}
$$

for both $i$ (cf. footnote 5 ).

This dynamic game can be represented by an Edgeworth box, in which the axes correspond with the two stages $t=1,2$ and at each stage, a total amount of 10 is distributed between the two agents (Figure 1). Although in principle allocations with negative amounts are allowed these do not play a role here.

Classical core. The classical core corresponds with the efficient and individually rational allocation streams in the Edgeworth box. The efficient allocation streams are the streams $\mathbf{x}=\left(\left(x_{1}^{1}, 10-x_{1}^{1}\right),\left(x_{1}^{2}, 10-x_{1}^{2}\right)\right)$ with $x_{1}^{1}=x_{1}^{2}$, represented by the diagonal line in Figure 1 . The individual rationality constraints are given by

$$
x_{1}^{1} x_{1}^{2} \geq 16 \text { and }\left(10-x_{1}^{1}\right)\left(10-x_{1}^{2}\right) \geq 16,
$$




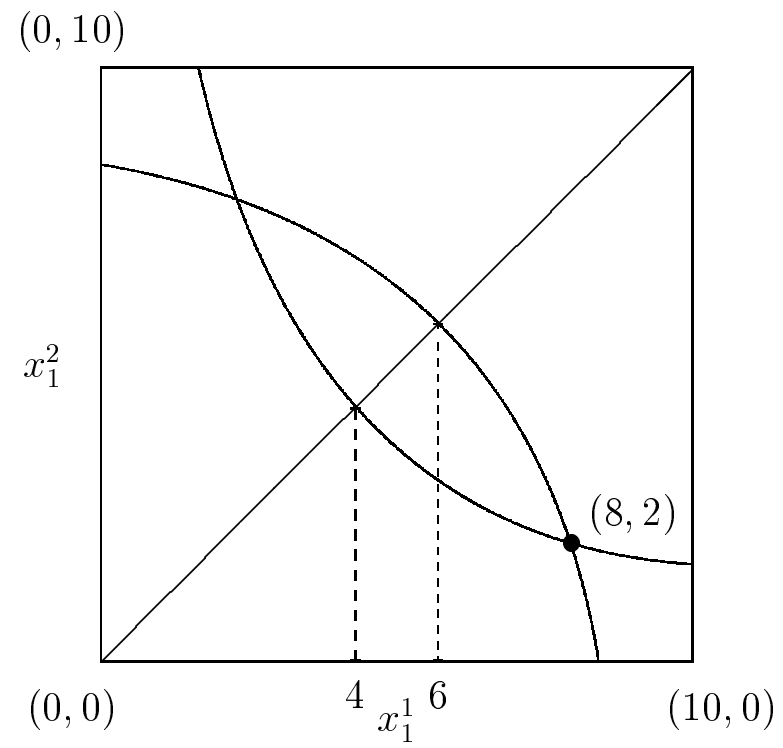

Figure 1: The Edgeworth box in Example 4

represented by the indifference curves in Figure 1. Therefore,

$$
C(\Gamma)=\{((x, 10-x),(x, 10-x)): 4 \leq x \leq 6\} .
$$

Weak sequential core. Every allocation stream $\mathbf{x}=\left(\left(x_{1}^{1}, x_{2}^{1}\right),\left(x_{1}^{2}, x_{2}^{2}\right)\right)$ in the weak sequential core has the property that $\left(x_{1}^{2}, x_{2}^{2}\right)=(2,8)$, since otherwise, either coalition $\{1\}$ or coalition $\{2\}$ could credibly deviate at the last stage. But then, also $\left(x_{1}^{1}, x_{2}^{1}\right)=(8,2)$, since otherwise either coalition $\{1\}$ or coalition $\{2\}$ could credibly deviate from $\mathbf{x}$ at the first stage. For instance, if $x_{1}^{1}<8$, then player 1 can credibly deviate at stage 1 since he can obtain $(8,2)$ by himself at stage 1 and 2 respectively, which is better than $\left(x_{1}^{1}, 2\right)$ obtained in $\mathbf{x}$. Hence, the only candidate for a weak sequential core allocation is $((8,2),(2,8))$.

We show that $\mathbf{x}=((8,2),(2,8))$ is indeed in the weak sequential core. It is clear that there is no credible deviation at the last stage. Suppose that there would be a credible deviation at the first stage. Since players 1 and 2 on their own can never improve upon $\mathbf{x}$, the only possibility remaining is that $\{1,2\}$ could credibly deviate at stage 1 . Let $\mathbf{y}=\left(\left(y_{1}^{1}, y_{2}^{1}\right),\left(y_{1}^{2}, y_{2}^{2}\right)\right)$ be such a credible deviation. Then, $\left(y_{1}^{2}, y_{2}^{2}\right)=(2,8)$, since otherwise either $\{1\}$ 
or $\{2\}$ could credibly deviate from $\mathbf{y}$ at the last stage. However, this implies that $\mathbf{y}$ cannot Pareto dominate $\mathbf{x}$, contradicting the fact that $\mathbf{y}$ is a credible deviation for $\{1,2\}$. Hence,

$$
W S C(\Gamma)=\{((8,2),(2,8))\}
$$

In particular, the classical core and the weak sequential core are both nonempty, but have an empty intersection.

Strong sequential core. Since the strong sequential core is contained in both the classical core and the sequential core, the strong sequential core is empty.

Example 5 For the classical core we have seen that, if the stage games all have a nonempty core and the utility functions are nicely behaved, then the classical core of the dynamic TU-game is nonempty. In this example we show that this result is no longer true for the weak sequential core (and therefore neither for the strong sequential core). Let $N=\{1,2,3\}$ and let $T=2$. Let the stage games be given by

$$
\begin{aligned}
v^{1}(\{i\}) & =0 \text { for } i=1,2,3, v^{1}(\{i, j\})=2 \text { for } i \neq j, v^{1}(\{1,2,3\})=3 ; \\
v^{2} & =v^{1} .
\end{aligned}
$$

Let the utility functions be given by

$$
\begin{aligned}
& u_{1}(a, b)=a b, \\
& u_{2}(a, b)=a b, \\
& u_{3}(a, b)=a b^{2} .
\end{aligned}
$$

Hence, both stage games have a nonempty core and Theorem 2 applies (cf. footnote 5.) We show, however, that the weak sequential core is empty.

Suppose that $\mathbf{x}=\left(x^{1}, x^{2}\right)$ would be a weak sequential core element. Then, $x^{2}=(1,1,1)$, since otherwise some two-person coalition would obtain less than 2, and this coalition could then credibly deviate at the last stage. But then, necessarily, $x^{1}=(1,1,1)$, since otherwise, some twoperson coalition would obtain less than 2 at the first stage, and this coalition could then credibly deviate at stage 1 . However, $\{1,3\}$ can credibly deviate from $((1,1,1),(1,1,1))$ at stage 1 by choosing a Pareto optimal allocation $\left(\left(y_{1}^{1}, y_{3}^{1}\right),\left(y_{1}^{2}, y_{3}^{2}\right)\right)$ dominating $((1,1),(1,1))$. Hence, the weak sequential core is empty. 


\subsection{Symmetric linear preferences}

In this subsection, the preferences are assumed to be symmetric and linear. Specifically, every player $i$ attaches utility $x_{i}^{1}+\cdots+x_{i}^{T}$ to a payoff stream $\mathbf{x}_{i}=\left(x_{i}^{1}, \ldots, x_{i}^{T}\right)$. A dynamic TU-game can therefore be denoted by $\Gamma=\mathbf{v}$.

In this case, for the classical core of $\Gamma$, we simply have $C(\Gamma)=C(v)$, where $v$ is the TU-game $v=\sum_{t=1}^{T} v^{t}$.

Before continuing we introduce a few notations. For a collection of coalitions $\mathcal{B} \subseteq 2^{N} \backslash\{\emptyset\}$, a coalition $S \subseteq N$ and a game $(N, v)$ let

$$
\begin{aligned}
C_{\mathcal{B}}(S, v):= & \left\{x \in \mathbb{R}_{+}^{S}: x(S)=v(S) \text { and } x(T) \geq v(T)\right. \\
& \text { for every } T \subseteq S \text { with } T \in \mathcal{B}\} .
\end{aligned}
$$

For a dynamic game $\mathbf{v}$ and $t=2, \ldots, T$ define

$$
\mathcal{B}^{t}:=\left\{S \in 2^{N} \backslash\{\emptyset\}: W S C\left(S, \mathbf{v}^{t}\right) \neq \emptyset\right\} .
$$

The following lemma characterizes the weak sequential core of a game $\mathbf{v}$.

Lemma 3 Let $\mathbf{v}$ be a dynamic game and let $\mathbf{x}=\left(x^{1}, \ldots, x^{T}\right)$ be a feasible allocation stream for the grand coalition $N$. Then $\mathbf{x} \in W S C(\mathbf{v})$ if and only if $\mathbf{x}^{t+1} \in W S C\left(\mathbf{v}^{t+1}\right)$ and $x^{t}+\cdots+x^{T} \in C_{\mathcal{B}^{t+1}}\left(N, v^{t}+\cdots+v^{T}\right)$ for every $t=1, \ldots, T-1$.

Proof. First suppose $\mathbf{x} \in W S C(\mathbf{v})$. By definition of the weak sequential core it follows that $\mathbf{x}^{t+1} \in W S C\left(\mathbf{v}^{t+1}\right)$ for every $t=1, \ldots, T-1$ (cf. Lemma 1 ). Let $t \in\{1, \ldots, T-1\}$ fixed and suppose, contrary to what we wish to prove, that $x^{t}(S)+\cdots+x^{T}(S)<v^{t}(S)+\cdots+v^{T}(S)$ for some $S \in \mathcal{B}^{t+1}$. Choose an arbitrary allocation stream $\left(y^{t+1}, \ldots, y^{T}\right) \in W S C\left(S, \mathbf{v}^{t+1}\right)$. For every $i \in S$ define $y_{i}^{t}:=x_{i}^{t}+\sum_{s=t+1}^{T}\left(x_{i}^{s}-y_{i}^{s}\right)+\alpha$ where $\alpha$ is chosen such that $y^{t}(S)=v^{t}(S)$, hence $\alpha|S|=\sum_{s=t}^{T} y^{s}(S)-\sum_{s=t}^{T} x^{s}(S)=\sum_{s=t}^{T} v^{s}(S)-$ $\sum_{s=t}^{T} x^{S}(S)>0$. If $\mathbf{y}^{t}=\left(y^{t}, \ldots, y^{T}\right)$ is a credible deviation for $S$ at stage $t$, then we are done because this contradicts $\mathbf{x} \in W S C(\mathbf{v})$. Otherwise, there is a coalition $S^{\prime} \subset S$ which can credibly deviate from $\mathbf{y}^{t}$ through an allocation stream $\mathbf{z}^{t}$. Since $\sum_{s=t}^{T} z_{i}^{s} \geq \sum_{s=t}^{T} y_{i}^{s}>\sum_{s=t}^{T} x_{i}^{s}$ for all $i \in S^{\prime}$ the coalition $S^{\prime}$ would have a credible deviation from $\mathbf{x}^{t}$ as well, again a contradiction (cf. Lemma 1).

Next, suppose that the allocation stream $\mathbf{x}$ satisfies the conditions in the lemma. Then a credible deviation can only be possible at $t=1$ for a coalition 
$S$ for which $W S C\left(S, \mathbf{v}^{2}\right) \neq \emptyset$, i.e., $S \in \mathcal{B}^{2}$. For such a coalition, however, $x^{1}(S)+\cdots+x^{T}(S) \geq v^{1}(S)+\cdots+v^{T}(S)$, implying that by deviating this coalition can never improve. Therefore $\mathbf{x} \in W S C(\mathbf{v})$.

The allocation $y^{t}$ for coalition $S$ constructed in the proof of Lemma 3 may have negative components: some players may accept a negative momentary payoff at time $t$ because they are compensated later.

Direct reference to the weak sequential cores of subgames can be avoided, as follows.

Theorem 5 Let $\mathbf{v}$ be a dynamic game and let $\mathbf{x}=\left(x^{1}, \ldots, x^{T}\right)$ be a feasible allocation stream for the grand coalition $N$. Then $\mathbf{x} \in W S C(\mathbf{v})$ if and only if $x^{T} \in C\left(v^{T}\right)$ and $x^{t}+\cdots+x^{T} \in C_{\mathcal{B}^{t+1}}\left(N, v^{t}+\cdots+v^{T}\right)$ for every $t=$ $1, \ldots, T-1$.

Proof. Every condition of the form $\mathbf{x}^{t} \in W S C\left(\mathbf{v}^{t}\right)$ occurring in Lemma 3 can, by the same lemma, be unraveled into the conditions $\mathbf{x}^{s} \in W S C\left(\mathbf{v}^{s}\right)$ for every $s=t+1, \ldots, T$ together with $x^{t}+\cdots+x^{T} \in C_{\mathcal{B}^{t+1}}\left(N, v^{t}+\cdots+v^{T}\right)$. By this the theorem follows.

The following lemma provides a recursive definition of the collection $\mathcal{B}^{t}(t=$ $2, \ldots, T)$ which, moreover, avoids reference to the weak sequential core.

Lemma 4 Let $\mathbf{v}$ be a dynamic game. Then for every $t=2, \ldots, T-1$ :

$$
\mathcal{B}^{t}=\left\{S \in \mathcal{B}^{t+1}: C_{\mathcal{B}^{t+1}}\left(S, v^{t}+\cdots+v^{T}\right) \neq \emptyset\right\},
$$

and

$$
\mathcal{B}^{T}=\left\{S \in 2^{N} \backslash\{\emptyset\}: C\left(S, v^{T}\right) \neq \emptyset\right\} .
$$

Proof. The statement about $\mathcal{B}^{T}$ is obvious. For $t=2, \ldots, T-1$ denote by $X^{t}(S)$ the set of allocations that are feasible for coalition $S$ at time $t$. We first prove the following

Claim: $\left\{S \in 2^{N} \backslash\{\emptyset\}\right.$ : there exists $\left(x^{t} \ldots, x^{T}\right) \in X^{t}(S) \times W S C\left(S, \mathbf{v}^{t+1}\right)$ with $\left.\sum_{\tau=t}^{T} x^{\tau} \in C_{\mathcal{B}^{t+1}}\left(S, v^{t}+\cdots+v^{T}\right)\right\}=\left\{S \in \mathcal{B}^{t+1}: C_{\mathcal{B}^{t+1}}\left(S, v^{t}+\cdots+v^{T}\right) \neq \emptyset\right\}$.

Proof of Claim: The inclusion from left to right is obvious. Now suppose that $C_{\mathcal{B}^{t+1}}\left(S, v^{t}+\cdots+v^{T}\right) \neq \emptyset$ and $W S C\left(S, \mathbf{v}^{t+1}\right) \neq \emptyset$. Choose $x \in C_{\mathcal{B}^{t+1}}\left(S, v^{t}+\right.$ 
$\left.\cdots+v^{T}\right)$ and $\tilde{\mathbf{y}}^{t+1} \in W S C\left(S, \mathbf{v}^{t+1}\right)$. Let $\tilde{\mathbf{y}}^{t+1}=\left(y^{t+1}, \ldots, y^{T}\right)$. Then $y^{\tau}(S)=$ $v^{\tau}(S)$ for all $\tau \geq t+1$, hence $\sum_{\tau=t+1}^{T} y^{\tau}(S)=\sum_{\tau=t+1}^{T} v^{\tau}(S)$. On the other hand, $x(S)=\sum_{\tau=t}^{T} v^{t}(S)$, so that $x(S)-\sum_{\tau=t+1}^{T} y^{\tau}(S)=v^{t}(S)$. Let $\tilde{\mathbf{x}}=$ $\left(x-\sum_{\tau=t+1}^{T} y^{\tau}, y^{t+1}, \ldots, y^{T}\right) \in X^{t}(S) \times W S C\left(S, \mathbf{v}^{t+1}\right)$. Then $\sum_{\tau=t}^{T} \tilde{x}_{i}^{\tau}=x_{i}$ so that $\sum_{\tau=t}^{T} \tilde{x}^{\tau} \in C_{\mathcal{B}^{t+1}}\left(S, v^{t}+\cdots+v^{T}\right)$. This completes the proof of the claim.

Now

$$
\begin{aligned}
\mathcal{B}^{t}= & \left\{S \in 2^{N} \backslash\{\emptyset\}: W S C\left(S, \mathbf{v}^{t}\right) \neq \emptyset\right\} \\
= & \left\{S \in 2^{N} \backslash\{\emptyset\}: \text { there exists }\left(x^{t} \ldots, x^{T}\right) \in\right. \\
& X^{t}(S) \times W S C\left(S, \mathbf{v}^{t+1}\right) \text { with } \\
& \left.\sum_{\tau=t}^{T} x^{\tau} \in C_{\mathcal{B}^{t+1}}\left(S, v^{t}+\cdots+v^{T}\right)\right\} \\
= & \left\{S \in \mathcal{B}^{t+1}: C_{\mathcal{B}^{t+1}}\left(S, v^{t}+\cdots+v^{T}\right) \neq \emptyset\right\}
\end{aligned}
$$

where the first and last equalities are by definition and the second equality follows from the Claim.

The following theorem presents a description of the strong sequential core. The simple (cf. Remark 1) proof is omitted.

Theorem 6 Let $\mathbf{v}$ be a dynamic TU-game and let $\mathbf{x}$ be an allocation stream. Then $\mathbf{x} \in S S C(\mathbf{v})$ if, and only if, $x^{t}+\cdots+x^{T} \in C\left(v^{t}+\cdots+v^{T}\right)$ for every $t=1, \ldots, T$.

\subsection{Lexicographic preferences}

In this subsection for simplicity we confine ourselves to the case $T=2$ and we assume that the players have lexicographic preferences. More precisely, we assume that every player $i$ either prefers the first or the second period. If he prefers the first period then he prefers $\left(x^{1}, x^{2}\right)$ to $\left(y^{1}, y^{2}\right)$ if either $x_{i}^{1}>y_{i}^{1}$ or $x_{i}^{1}=y_{i}^{1}$ and $x_{i}^{2} \geq y_{i}^{2}$. The preference of a player $i$ who prefers the second period is defined similarly, with the roles of the two periods reversed. These preferences are not even representable by utility functions but nevertheless the definitions of classical and strong and weak sequential cores should be obvious.

We assume in this subsection that only nonnegative allocations are possible. Without this assumption (or at least an assumption that allocations 
are bounded from below) the classical core would be empty if both types of players are present: in period one we can have unlimited transfers from players who prefer period two to players who prefer period one, provided we have the reverse transfers in period two. Hence, any allocation can be blocked by a better one. By a similar argument it might also be the case that the number of credible deviations is reduced but, in general, the consequences of allowing unbounded allocations for the weak sequential core are less clear.

Assume that $\left(v^{1}, v^{2}\right)$ is a two-period dynamic TU-game with lexicographic preferences. For every coalition $S$ write $S=S^{1} \cup S^{2}$ where $S^{i}$ consists of those players that prefer period $i$. Let $\left(x^{1}, x^{2}\right)$ be a feasible allocation stream for the grand coalition $N$. Then we have:

Theorem 7 The following two statements are equivalent:

(a) $\left(x^{1}, x^{2}\right) \in W S C\left(v^{1}, v^{2}\right)$,

(b) $x^{2} \in C\left(v^{2}\right)$ and for all coalitions $S=S^{1} \cup S^{2}$ and all $y^{2} \in C\left(S, v^{2}\right)$, if $y_{i}^{2} \geq x_{i}^{2}$ for all $i \in S^{2}$, then $\sum_{i \in S^{2}: y_{i}^{2}=x_{i}^{2}} x_{i}^{1} \geq v^{1}(S)-x^{1}\left(S^{1}\right)$.

Proof. First assume $\left(x^{1}, x^{2}\right) \in W S C\left(v^{1}, v^{2}\right)$. Then $x^{2} \in C\left(v^{2}\right)$ (cf. Lemma 1). Suppose $S=S^{1} \cup S^{2}$ and $y^{2} \in C\left(S, v^{2}\right)$ with $y_{i}^{2} \geq x_{i}^{2}$ for all $i \in$ $S^{2}$. Then consider any feasible allocation $\left(z^{1}, y^{2}\right)$ for $S$ with $z_{i}^{1}>x_{i}^{1}$ for all $i \in S^{1}$. By Lemma 1 it must be the case that at least one player in $S^{2}$ is worse off than at $\left(x^{1}, x^{2}\right)$. This implies the condition in (b), viz., $\sum_{i \in S^{2}: y_{i}^{2}=x_{i}^{2}} x_{i}^{1} \geq v^{1}(S)-x^{1}\left(S^{1}\right)$. For the implication (b) $\Rightarrow(\mathrm{a})$, if $x^{2} \in C\left(v^{2}\right)$ and $x^{1}\left(S^{1}\right) \geq v^{1}(S)$ for all $S$, then (a) follows by Lemma 1. If $x^{1}\left(S^{1}\right)<v^{1}(S)$ for some $S$ then any improvement for $S^{1}$ must distribute at least $x^{1}\left(S^{1}\right)$ in $v^{1}$ among the players of $S^{1}$, but then in any element in $C\left(S, v^{2}\right)$, by the implication in (b) at least one player in $S^{2}$ must be worse off.

The classical core of the two-stage game with lexicographic preferences is characterized in the following theorem. The proof is left to the reader.

Theorem 8 The following two statements are equivalent:

(a) $\left(x^{1}, x^{2}\right) \in C\left(v^{1}, v^{2}\right)$,

(b) for all coalitions $S=S^{1} \cup S^{2}$ we have 
(i) either $x^{1}\left(S^{1}\right)>v^{1}(S)$

(ii) or $x^{1}\left(S^{1}\right)=v^{1}(S)$ and $x^{2}(S) \geq v^{2}(S)$

(iii) or $x^{1}\left(S^{1}\right)<v^{1}(S)$ and $x^{2}\left(S^{2}\right)>v^{2}(S)$

(iv) or $x^{1}\left(S^{1}\right)<v^{1}(S)$ and $x^{2}\left(S^{2}\right)=v^{2}(S)$ and $x^{1}(S) \geq v^{1}(S)$.

As usual (cf. Remark 1) the strong sequential core is the subset of the (classical) core consisting of those $\left(x^{1}, x^{2}\right)$ with $x^{2} \in C\left(v^{2}\right)$.

This part is conluded with a simple example.

Example 6 Let $N=\{1,2\}$ and let $v^{1}=v^{2}$ be given by $v^{1}(\{i\})=v^{2}(\{i\})=$ 1 for $i=1,2$ and $v^{1}(N)=v^{2}(N)=10$. Suppose player 1 prefers $t=1$ and player 2 prefers $t=2$. Then $C\left(v^{1}, v^{2}\right)=\{(10,0),(0,10)\}$, and

$$
\begin{aligned}
W S C\left(v^{1}, v^{2}\right)= & \left\{\left(x^{1}, x^{2}\right) \geq 0: x_{1}^{1}+x_{2}^{1}=10, x_{1}^{2}+x_{2}^{2}=10,\right. \text { and } \\
& \text { either } \left.x_{1}^{2}=1 \text { and } x_{1}^{1} \geq 1 \text { or } 1<x_{1}^{2}<9 \text { and } x_{1}^{1}=10\right\} .
\end{aligned}
$$

From this (or directly) it follows that the strong sequential core of this game is empty.

\section{Concluding remarks}

In this paper we have proposed several core concepts for dynamic cooperative finite horizon games, where at each moment a transferable utility game is played. For the strong and weak sequential cores of such games the emphasis was on the definitions and examples as well as special cases, since it is not obvious whether general and useful existence results can be derived. One interesting avenue for continued research is the application of, in particular, the weak sequential core concept to other models. Gale (1978, footnote 9 ), for example, hints at such an application without further exploring the subject.

The appropriateness of a particular core concept for a dynamic cooperative game depends on the nature of the contracts that can be written in that particular situation. Roughly, the classical core is appropriate in a situation where at the beginning of the game a complete contract on the allocation stream is possible. In a world of more or less incomplete contract possibilities other concepts such as the sequential cores may be more appropriate. 
This paper is part of a more general research project that aims at developing solution concepts for dynamic cooperative games. Other work may concentrate on value-like solutions, such as the Shapley value. A further interesting avenue in this project deals with the situation where the stage cooperative game depends on the history of play, as studied in Filar and Petrosjan (2000). These topics will be subject of future research.

\section{References}

Becker, R.A. and S.K. Chakrabarti (1995): "The Recursive Core," Econometrica, 63, 401-423.

Bondareva, O.N. (1963): "Some Applications of Linear Programming Methods to the Theory of Cooperative Games," (in Russian) Problemy Kibernetiki, $10,119-139$.

Filar, J.A., and L.A. Petrosjan (2000): "Dynamic Cooperative Games," International Game Theory Review, 2, 47-65.

Gale, D. (1978): "The Core of a Monetary Economy without Trust," Journal of Economic Theory, 19, 456-491.

Kannai, Y. (1992): "The Core and Balancedness," in: Handbook of Game Theory with Economic Applications, Chapter 12 (eds. R.J. Aumann and S. Hart). Amsterdam: North-Holland.

Koopmans, T.C. (1960): "Stationary Ordinal Utility and Impatience," Econometrica, 28, 287-309.

Koutsougeras, L.C. (1998): "A Two-Stage Core with Applications to Asset Market and Differential Information Economies," Economic Theory, 11, 563584 .

Munro, G. (1979): "The Optimal Management of Transboundary Renewable Resources," Canadian Journal of Economics 12, 355-376.

Predtechenski, A., P.J.J. Herings, and H. Peters (2001): "The Strong Sequential Core for Two-Period Economies," Department of Economics, University of Maastricht.

Ray, D. (1989): "Credible Coalitions and the Core," International Journal of Game Theory, 18, 185-197. 
Rosenthal, E.C. (1990a): "Monotonicity of Solutions in Certain Dynamic Cooperative Games," Economics Letters, 34, 221-226.

Rosenthal, E.C. (1990b): "Monotonicity of the Core and Value in Dynamic Cooperative Games," International Journal of Game Theory 19, 45-57.

Scarf, H.E. (1967): "The Core of an n-Person Game," Econometrica, 35, $50-69$.

Shalev, J. (1995): "Loss Aversion and Bargaining," mimeo.

Shapley, L.S. (1967): "On Balanced Sets and Cores," Naval Research Logistics Quarterly, 14, 453-460.

Strotz, R.H. (1955): "Myopia and Inconsistency in Dynamic Utility Maximization," Review of Economic Studies, 23, 165-180. 